\title{
Thermo-Fluid Simulation for Indoor Air Quality and Buildings Thermal Comfort
}

\author{
Souad. Morsli ${ }^{1}$, ${ }^{2 *}$, Amina. Sabeur ${ }^{1}$, Harry. Ramenah ${ }^{2}$, Mohammed. El Ganaoui ${ }^{3}$, Rachid.Bennacer ${ }^{4}$ \\ ${ }^{1}$ University of Science and Technology Mohamed Boudiaf/LSIM .Oran Algeria. \\ ${ }^{2}$ University of Lorraine, LCOMS-Metz, France. \\ ${ }^{3}$ University of Lorraine, IUT / LERMAB-Longwy, France. \\ ${ }^{4}$ University Paris Saclay, LMT-ENS Cachan, France
}

Email: souad.morsli@yahoo.fr

\begin{abstract}
Air conditioning systems are seen as a crucial part of any structure because they are directly related to the comfort of the occupants. Finding a conditioning system that exhibits not only a high level of comfort, but also a decrease in energy consumption and cost of the system is now very much preferable, especially with the ever-increasing cost of energy. Mixed convection for different boundary conditions and different configuration is carried out. In addition, a flow is injected through a window and extracted through an opposite window. In this work we examine the influence of the value of the Rayleigh number on the flow structure. The numerical analysis was carried out using the 'Fluent' software. To deal with turbulence the RNG k- $\varepsilon$ model was adopted in this study. The study of ventilation efficiency has shown that the intensity of the recirculation flow increases near the adiabatic wall with the increase of Rayleigh number.
\end{abstract}

Keywords: Energy consumption; mixed convection; ventilation efficiency.

\section{Nomenclature}

$\begin{array}{llr}\mathrm{g} & \text { gravitational acceleration } & {\left[\mathrm{m} \mathrm{s}^{-2}\right]} \\ \mathrm{L}_{\mathrm{x}} & \text { dimension of cavity in } \mathrm{x} \text { direction } & {[\mathrm{m}]} \\ \mathrm{L}_{\mathrm{y}} & \text { dimension of cavity in y direction } & {[\mathrm{m}]} \\ \mathrm{L}_{z} & \text { dimension of cavity in } \mathrm{z} \text { direction } & {[\mathrm{m}]} \\ \mathrm{T} & \text { dimensional temperature } & {[\mathrm{K}]} \\ \mathrm{u}, \mathrm{v}, \mathrm{w} & \text { components of dimensionless velocity } & \\ \mathrm{x}, \mathrm{y}, \mathrm{z} & \text { dimensionless Cartesian coordinates } & \end{array}$

Greek Symbols

\begin{tabular}{|c|c|c|c|c|c|}
\hline$\alpha$ & thermal diffusivity & {$\left[\mathrm{m}^{2} \mathrm{~s}^{1}\right]$} & $\mathrm{Nu}_{\text {Lat }}$ & $N u$ on lateral walls & $=\int^{1} \int^{1} \frac{\partial \theta}{\partial x} d y d x$ \\
\hline$\beta_{\mathrm{T}}$ & coefficient of volumetric expansion & {$\left[\mathrm{K}^{1}\right]$} & & & $\left.x\right|_{z=0,1}$ \\
\hline$\mu$ & dynamic viscosity & {$\left[\mathrm{kg} \mathrm{m}^{1} \mathrm{~s}\right]$} & $\operatorname{Pr}$ & Prandtl number, & $=v / \alpha$ \\
\hline$v$ & kinematic viscosity & {$\left[\mathrm{m}^{2} \mathrm{~s}^{1}\right]$} & $\mathrm{Ra}$ & Rayleigh number, & $=g \beta_{T} \Delta T L_{Z}^{3} /(v \alpha)$ \\
\hline$\rho$ & mass density & {$\left[\mathrm{kg} / \mathrm{m}^{3}\right]$} & & & \\
\hline$\theta$ & dimensionless temperature, & $\mathrm{C}) /\left(\mathrm{T}_{\mathrm{H}}-\mathrm{T}_{\mathrm{C}}\right)$ & & & \\
\hline
\end{tabular}

Non-dimensional Numbers

$\mathrm{A}_{\mathrm{x}} \quad \mathrm{x}$ aspect ratio, $\quad L_{x} / L_{z}$

$\mathrm{A}_{\mathrm{y}} \quad \mathrm{y}$ aspect ratio, $\quad L_{y} / L_{z}$

$\mathrm{h}, \mathrm{d}$ dimensionless ventilation window, scaled

$\mathrm{Nu}_{\text {down }} \quad \mathrm{Nu}$ on bottom wall $\quad=\left.\int_{y=0}^{1} \int_{z=0}^{1} \frac{\partial \theta}{\partial x}\right|_{x=0} d y d z$ $=0$ 


\section{Introduction}

In a context of control of energy demand, the building sector is taking a relatively larger share in total French energy consumption. Many studies are conducted to improve the performance of buildings to reduce energy dependence and ensure thermal comfort of occupants. For this purpose, thermal regulations and / or several labels have been introduced to limit the energy consumption for the standard use of the building: heating, air conditioning, ventilation and lighting.

In France, these thermal regulations apply for all buildings including the tertiary sector (commercial, industrial, building receiving public), with three major requirements: (1) the improvement of the energy efficiency, (2) the reduction of energy consumption and (3) summer comfort in non-air-conditioned buildings (RT2012, 2012). The tertiary sector can present many problems of thermal discomfort and high energy consumption both in winter (for heating) and summer (for cooling). Especially nowadays, man spends most of his time in closed spaces: comfort becomes an important parameter for the well-being of the occupants; indoor air is a major concern for people and public authorities.

Homes, schools and leisure facilities, office space, transportation, are so much life places where many substances and backgrounds agents are often present at concentrations higher than those measured in the outside air. The factors involved are numerous: presence and human activity, materials, furniture and systems equipping buildings, outdoor environment nearby, etc.

The degradation of the quality of indoor air is a problem that has attracted major interest in our industrialized societies, and has been for several years.

Among the first studies on the mixed convection in ventilated cavities, we mention that undertaken by Baines and Turner [1] in closed space. In the case of opening cavity, Woods et al. [2] shows the existence of two main regimes (displacement and blocked regimes) of ventilation by changing the rate of flow. The same regimes are observed by Allano et al. [3], by changing the dimension of the opening zone. Papanicolaou and jaluria [4] presented numerically the influence of the thermal conductivity, Richardson and Reynolds numbers on the average Nusselt number induced by mixed convection within enclosure. Overall, they find that the heat transfer increase with increasing this controlling parameters. By adding a heat sources in the ventilated rectangular cavity, the same authors Papanicolaou and jaluria [5] showed that the location of the sources strongly influences the flows structures, and an oscillatory behavior was obtained for $\mathrm{Gr} / \mathrm{Re}^{2}=50$. The influence of this parameter $\left(\mathrm{Gr} / \mathrm{Re}^{2}\right)$ on the heat transfer is also demonstrated by Hsu and Wang [6] in the case of mixed convection within cavity with two ventilation ports. Raji et al. [7] studied the mixed convection heat transfer in a ventilated cavity. The numerical results show the presence of a maximum interaction between the effects of the forced and natural convection, with the existence of different flow regimes, delineated in the Ra-Re plane. Bhoite et al. [8], deals with the problem of mixed convection in shallow enclosure with series of heat generating components; they shown that higher Reynolds number tend to create a recirculation region of increasing strength at the core region and that the effect of buoyancy becomes insignificant beyond $\mathrm{Re}=600$. On the basis of the literature review, it appears that several investigations (Calmidi and Mahajan [9], Singh and Sharif [10]) have analyzed a number of governing parameters in natural or mixed convection in closed or open cavities, including thermal sources, fluid property, supplying fluid velocity and dimension or location of ports. In the case of closed cavity, no work was reported on mixed convection in ventilated enclosure induced by internal inertial point (or not) sources. Indeed, the existence of a dynamic internal source will create interaction with the thermal forces, induced by differential heating; causing the birth of mixed flow.

We consider in this work a room of a housing consisting of a cooling floor. This floor is maintained at a constant cold temperature and the one vertical wall of the room is kept at a hot temperature and the rest of the walls are adiabatic. We focus on the influence of various physical and geometrical parameters on the flow structures that can occur and on the heat transfer exchange.

\section{Problem Definition \& Governing Equations}

The configuration studied is a cubic cavity filled with air $\operatorname{Pr}=0.71$ (Fig.3). The dimensions of the cavity are $\mathrm{L}_{\mathrm{x}}$, $\mathrm{L}_{\mathrm{y}}$ and $\mathrm{L}_{\mathrm{z}}$. The temperature of the floor is maintained at a temperature $T_{c}$ and the temperature of the left vertical wall at $T_{h}$. Different scenaris are envisaged for different thermal conditions on the walls. Other bonding walls are assumed to be adiabatic. We inject air at the same temperature on the heated side from a small window and extract the same amount of air from a similar window on the opposite side.

The air is supposed to be Newtonian and incompressible and satisfies Boussinesq's hypothesis. On the one hand, we are interested in the influence of ventilation and heating on the dynamics of the flow and the heat transfer taking into account the Ra number which varies as a function of the heating temperature.

\section{Mathematical Formulation}

Using the following dimensionless variables:

$X=\frac{x}{L_{x}}, Y=\frac{y}{L_{x}}, Z=\frac{z}{L_{x}}, P=\frac{p}{\rho v^{2}}, \vec{V}=\frac{\vec{v} L_{x}}{v}, \theta=\frac{T-T_{c}}{T_{h}-T_{c}}$

Where $v$ is the kinematical viscosity of the fluid and $\overrightarrow{\mathrm{v}}$ is the velocity vector.

The equations governing the conservation of mass, momentum and energy in non dimensional form can be written as, 


$$
\begin{aligned}
& \vec{\nabla} \cdot \vec{V}=\mathrm{O} \\
& (\vec{V} \cdot \vec{\nabla}) \vec{V}=-\vec{\nabla} P+\nabla^{2} \vec{V}+\frac{R a}{\operatorname{Pr}} \theta \overrightarrow{\boldsymbol{k}} \\
& \vec{V} \cdot \nabla \theta=\frac{1}{\operatorname{Pr}} \nabla^{2} \theta
\end{aligned}
$$

Where $\overrightarrow{\boldsymbol{k}}$ the unit vector in $\mathrm{Z}$ direction is, $R a=\frac{g \beta_{T} \Delta T L_{x}{ }^{3}}{v \alpha}$ is the thermal Rayleigh number and $\operatorname{Pr}=\frac{v}{\alpha}$ is the Prandtl number.

Boundary conditions for the velocities are no slip condition $(\mathrm{U}=\mathrm{V}=\mathrm{W}=0)$ on all solid boundaries;

$\mathrm{U}=\mathrm{Re}$ on $\mathrm{X}=0,1 \& 0.28<\mathrm{Y}<0.72 \& 0.71<\mathrm{Z}<0.86$

The thermal boundary conditions are:

The side wall at $\mathrm{X}=0$ is heated at $\theta=1$, and the wall at $\mathrm{Z}=0$ is cooled $\theta=0$, all other walls are adiabatic

$$
\partial \theta / \partial n=0
$$

RNG $\mathrm{k}-\varepsilon$ "turbulence model" leads to two additional expressions for the turbulence kinetic energy $\mathrm{k}$, and the dissipation rate, $\varepsilon$, respectively:

$$
\begin{aligned}
& \frac{\partial}{\partial x_{i}}\left(\rho x_{i} k\right)=\frac{\partial}{\partial x_{i}}\left(\alpha_{k} \mu_{e f f} \frac{\partial k}{\partial x_{i}}\right)+G_{k}-\rho \varepsilon \\
& \frac{\partial}{\partial x_{i}}\left(\rho x_{i} \varepsilon\right)=\frac{\partial}{\partial x_{i}}\left(\alpha_{\varepsilon} \mu_{e f f} \frac{\partial k}{\partial x_{i}}\right)+\frac{\varepsilon}{k}\left(C_{1 \varepsilon} G_{k}-C_{2 \varepsilon} \rho \varepsilon-\chi\right)
\end{aligned}
$$

\section{Numerical Tools}

Fluent industrial software [11] was chosen as a CFD computational package which. It is based on a finite-volume procedure to produce numerical solution of the NavierStokes set of fluid flow in primitive variables $(\mathrm{P}, \mathrm{V}, \mathrm{T})$. The Code provides also solutions for transport of chemical components by solving partial differential equations describing basic mechanisms as convection, diffusion, and reaction for each constituent. The RNG model was chosen for turbulence modeling [12].

The numerical resolution of the equations was performed on a 2D-3D dimensional configuration. The mesh used was realized on Gambit for the different configurations studied. The mesh has been refined close to the walls (presence of boundary layers with strong gradients).

The Finite Volumes method consists in discretizing the computational domain in sub-domains or control volumes whose faces follow the lines of coordinates. Fluent allows choosing the discretization schemes for different operators. A "segregated" solver that calculates each equation separately is chosen.

The pressure equation is discretized by the standard scheme. The interpolation is done using the coefficients of the equation of quantity of movement. This procedure works well for small variations in pressure between the centers of the cells. Strong pressure gradients between the cells generate inaccuracy on estimating the velocity on the faces. This is the case of a swirling flow at high speed generally the pressure is unknown and there is no obvious equation for its determination. The method used to solve this problem was developed by Van Doormal \& Raithby in 1984: it is the SIMPLEC algorithm (Semi-Implicit Method for Pressure Linked Equations-Consistent).

For the other diffusion convection equations (momentum, energy, turbulence ...), Quick interpolation schemes have been adopted.

\section{Simulation Results}

Since the interest is on the thermal comfort of using floor cooling. The temperature and the velocitycontours in the enclosure are presented and discussed for Rayleigh numbers of $10^{3}$, to $10^{6}$. The flow became unstable when Rayleigh numbers exceeds $10^{7}$. The results presented concern a two-dimensional and cubic enclosure. Figure1 show temperature field in the two- dimensional enclosure for $\mathrm{Ra}=10^{3}$ to $10^{7}$. The flow is thermally stratified in the core of the enclosure for adiabatic ceiling condition. The results show sharp gradient near the floor and gradually the temperature increases toward the ceiling. Also, temperature is almost uniform in planes parallel to the floor, except near the heated wall. The level of hot planes is driven down when Ra increases.

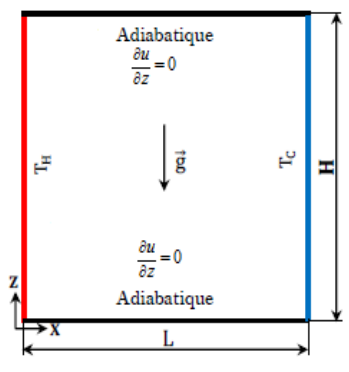

(a)

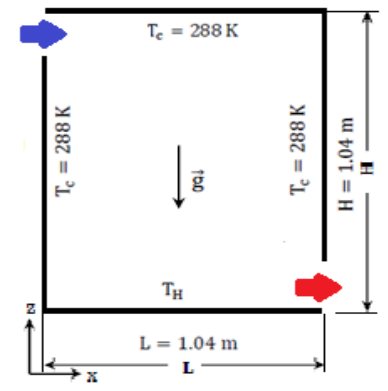

(b)
Fig.1. Physical model of the room.

Among the cases studied in this study, the closed cavity was chosen in a laminar regime (Fig. 1a) and the case where the room is ventilated under isothermal conditions under turbulent conditions (Fig. 1b). Compared to the results obtained from the experimental study of Krane et al [13], and the numerical study of Morsli [14] with the same parameters. A good agreement was generally found for both comparisons.

Fig.2 ( $a$ and $b$ ) show the comparison of the velocity profiles $\mathrm{u}$ and $\mathrm{w}$ obtained numerically and experimentally (Krane and [13] and Morsli [14]) at $Z^{*}=0.5$ and $X^{*}=0.5$ respectively. We note that the profiles of $u$ and $w$ are identical. We note that the maximum and minimum values $\mathrm{u}$ and $\mathrm{w}$ obtained by numerical simulation are slightly lower 
than those of Krane et al [13]. This is probably due to the mesh which remained coarse near the walls. Figure 2 (c) illustrates the profiles $\mathrm{T}$ to $\mathrm{Z}^{*}=0.5$. It is noted that the temperature is quasi-homogeneous in the middle of the cavity and verifies well its value reached at the level of the vertical walls.

On the whole, the agreement between the forecasts and the measures is very satisfactory. Figure 2 (d) illustrates the evolution of the local number of Nusselt $(\mathrm{Nu})$ calculated at the hot wall as a function of the height
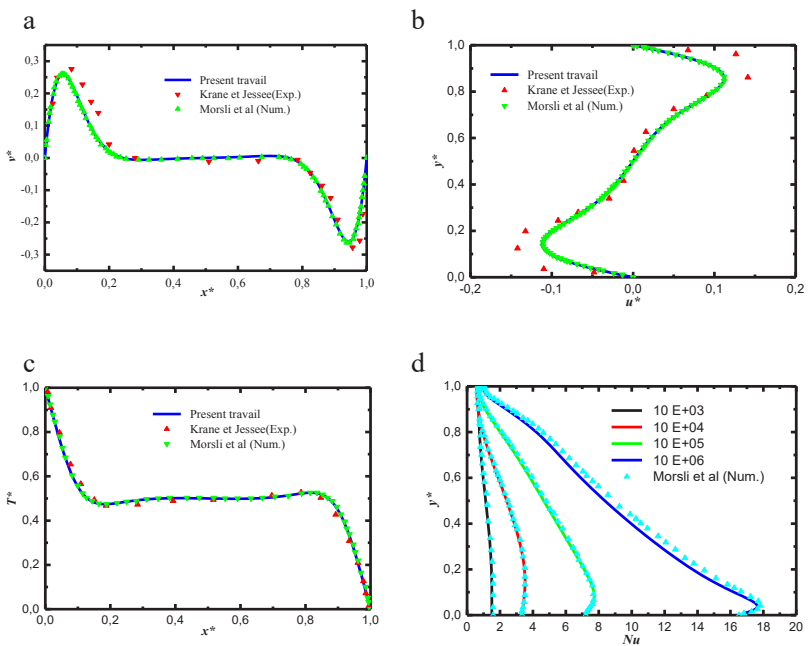

Fig. 2. Confrontation between the numerical results and those of ref. 13 and 14 for, (a) profile of $\mathrm{u}^{*}$, (b) profile of $\mathrm{w}^{*}$, (c) profile of $\mathrm{T}^{*}$; for $\mathrm{Ra}=1.89 .10^{5}$ and (d) Nu number evolution along the hot wall.

of the cavity, for different values of $\mathrm{Ra}$. The number $\mathrm{Nu}$ is greater in the vicinity of the lower horizontal wall $\left(Z^{*}=1\right)$. This translates into a maximum heat transfer rate due to the fresh air that heats near the hot wall. Consequently, its density decreases, it increases due to the strength of Archimedes and modifies its trajectory at the level of the upper horizontal wall. At $Z^{*}=1$, the heat transfer rate is minimal due to the hot air at the upper horizontal wall which tends to move towards the cold wall. This implies low $\mathrm{Nu}$ values.

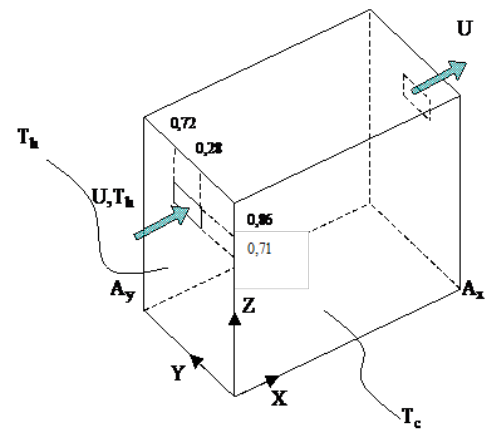

Fig.3. Configuration 3D studied.
Figure 4a shows the velocity profiles $u$ at $X^{*}=0.5$ and $Z^{*}$ $=0.5$. By changing from $\mathrm{Ra}_{\mathrm{T}}=10^{3}$ to $\mathrm{Ra}_{\mathrm{T}}=10^{4}$, the values of the velocities $u$ increase. Whereas, for $\operatorname{Ra}_{\mathrm{T}}>10^{4}$, they gradually decrease throughout the cavity.

Figure $4 \mathrm{~b}$ shows the velocity profile $\mathrm{w}$ at $\mathrm{Z}^{*}=0.5$ and at $X^{*}=0.5$ by varying $\mathrm{Ra}_{\mathrm{T}}$ from $10^{3}$ to $10^{4}$, the values of $\mathrm{w}$ increase. However, for $\operatorname{Ra}_{\mathrm{T}}>10^{4}$, w decreases progressively at the center of the cavity and becomes almost zero at $\mathrm{Ra}_{\mathrm{T}}=10^{6}$ and increases at the boundary layers. Observations from temperature profiles (figure 5) show that when convection takes effect in one area, boundary layers develop near the walls.

The transfer of heat through these boundary layers is only by conduction and the core of the vortex is homogenized to tend towards an almost constant temperature. Low and high temperatures lie within the boundary layers along the isothermal walls. The thickness of the boundary layers depends mainly on the intensity of convection i.e. of the value of the $\mathrm{Ra}_{\mathrm{T}}$ number.

The evolution of the isotherms inside the cavity is consistent with the circulation of the fluid revealed by the velocity fields. It has been found that the isotherms (Figure5) become more and tighter at the sidewalls as $\mathrm{Ra}_{\mathrm{T}}$ increases. To get a clear idea about the behavior of the flow and the heat transfer inside the cavity, we present the velocity fields and the isotherms (figure $5 \& 6$ ). We observe that near the ceiling $(\mathrm{Z}=1.04 \mathrm{~m})$, the jet remains parallel to the wall and when it hits the right vertical wall $(\mathrm{X}=1.04$ $\mathrm{m})$, it changes direction giving rise to vortex structures. The first so-called primary structure is a large vortex located in the center of the cavity.
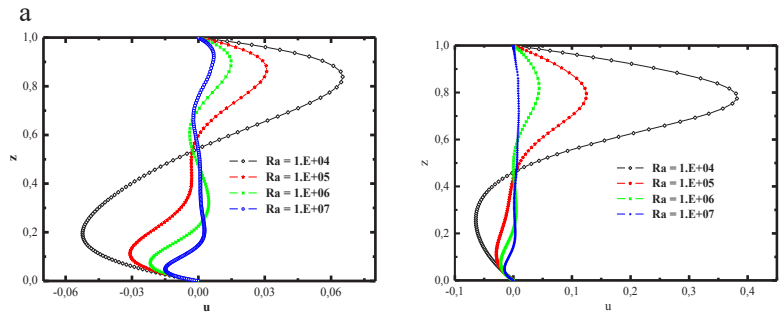

b
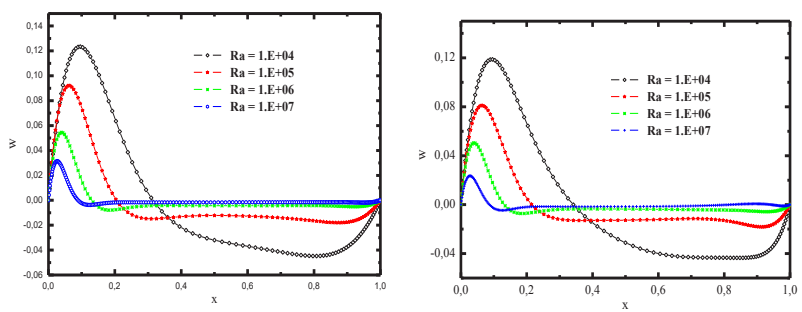

Fig.4. Velocity profiles $u$ and $w$ for different values of Ra: (a) $\mathrm{X}=0.5$, (b) $\mathrm{Z}=0.5$. closed cavity (right) and an open cavity (left) 
As for the secondary structures at the corners are of different sizes. Indeed, we notice two small whirlpools located near the entrance and at the upper right corner. With regard to the isotherms, we observe that the cold air heats up when it passes near the hot wall. Then, it branches off to go up to the fresh air entrance. During this movement the heat is brought to the center of the cavity. On the other hand, the increase in $\mathrm{Ra}_{\mathrm{T}}$ does not result in a change in flow behavior. in the case of the open cavity the velocity fields indicate that the flow intensifies as a function of the $\mathrm{Ra}_{\mathrm{T}}$ number for a given $\mathrm{Re}$, we notice the formation of a small structure $\left(\mathrm{Ra}_{\mathrm{T}}=10^{3}\right)$ along the left vertical wall.
$\mathrm{Ra}=10^{4}$

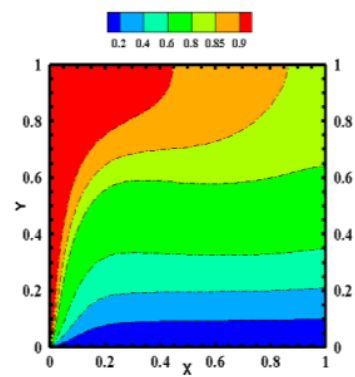

$\mathrm{Ra}=10^{6}$

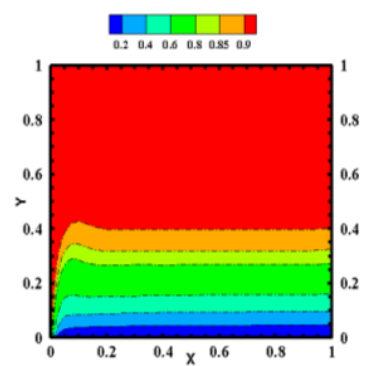

$\mathrm{Ra}=10^{7}$

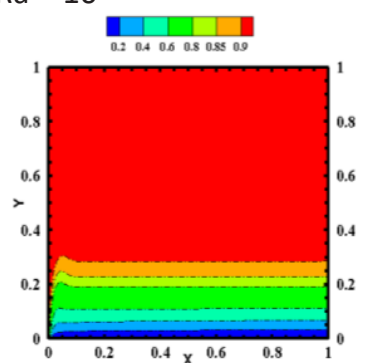

$\mathrm{Ra}=10^{4}$

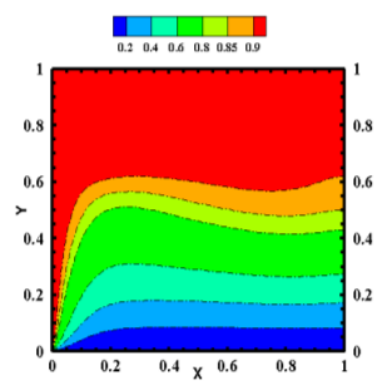

$\mathrm{Ra}=10^{6}$

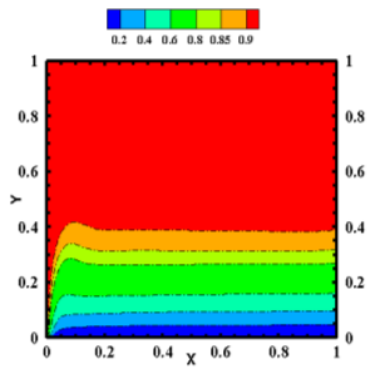

$\mathrm{Ra}=10^{7}$

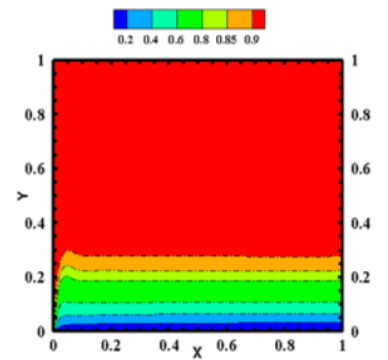

Fig.5. The temperature contours $\mathrm{T}$ for different Rayleigh numbers a closed cavity (right) and an open cavity (left).

This structure widens by increasing the $\mathrm{Ra}_{\mathrm{T}}$ number and occupies a good part of the cavity at $\mathrm{Ra}_{\mathrm{T}}=10^{5}$. At the level of the right vertical and horizontal lower walls, the flow is driven only by forced convection. Indeed, the air penetrates parallel to the lower horizontal wall, changes direction close to the right vertical wall and becomes parallel to it to reach the exit. we observe another structure that tends to disappear by increasing the number $\mathrm{Ra}_{\mathrm{T}}$. This is the transition structure

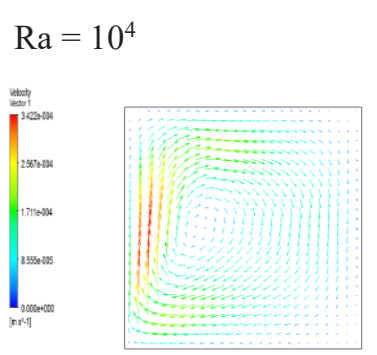

$\mathrm{Ra}=10^{4}$

$\mathrm{Ra}=10^{5}$

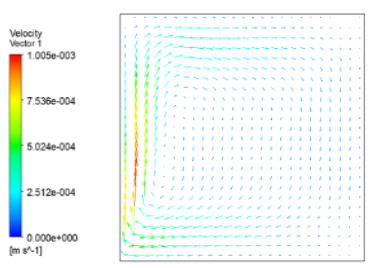

$\mathrm{Ra}=10^{5}$

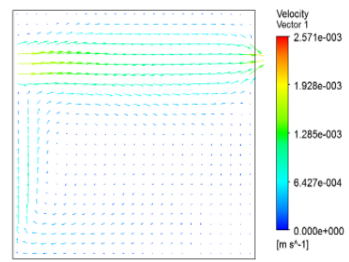

$\mathrm{Ra}=10^{6}$

$\mathrm{Ra}=10^{6}$
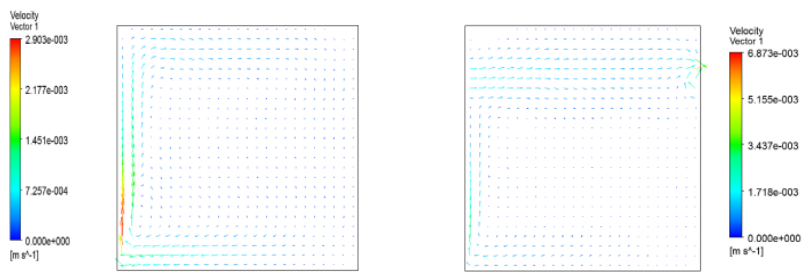

$\mathrm{Ra}=10^{7}$

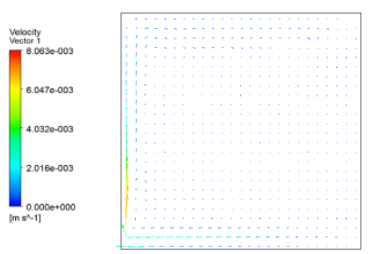

$\mathrm{Ra}=10^{7}$
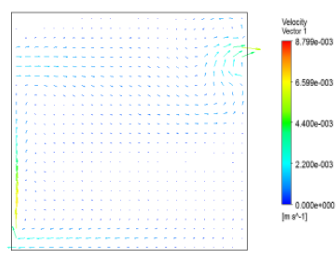

Fig. 6. Velocity fields for different Rayleigh Numbers a closed cavity (right) and an open cavity (left).

between natural convection and forced convection. Figures 7 shows that the temperature fields and the flow are thermally stratified in the center (core) of the cavity for the condition of adiabaticity of the ceiling for $\mathrm{Ra}=10^{4}$.

These results also show that the temperature gradients near the floor are low and the temperature rises near the ceiling. In addition, the temperature is almost uniform in planes parallel to the floor except near the hot wall. Stratification levels decrease with increasing number of Ra's. It has been found that the degree of stratification depends on the Rayleigh number. For the condition of a refreshed ceiling, the structure of the three-dimensional flow has been modified and leads to transverse recirculation towards the sides and downwards. Moreover, the intensity of the recirculation flow increases near the adiabatic wall 
with the Rayleigh number. Thermal comfort can be achieved by orienting the direction of air injection relative to the horizontal direction of the floor using a deflector.
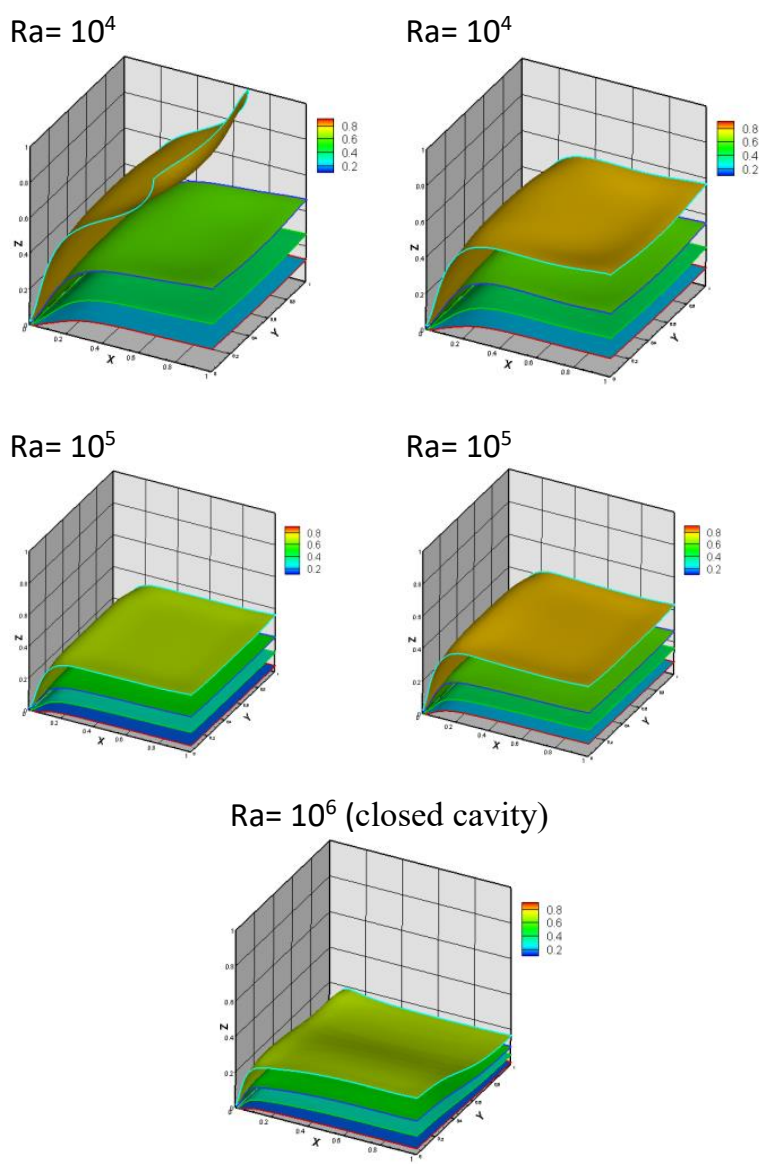

Fig.7. 3D temperature contours at $X^{*}=0.5$ and $Y^{*}=0.5$ for different Rayleigh Numbers a closed cavity (right) and an open cavity (left).

For values of $\mathrm{Ra}=10^{5}$, and $10^{6}$ we can see that the open cavity modifies the distribution of the temperature field and the structure of the flow.

In the horizontal direction to the cooling floor, the temperature is not homogeneous on the horizontal plane. In addition, the intensity of the recirculation flow was increased compared to the results obtained for $\mathrm{Ra}=10^{6}$ and causes the flow of air down, it is found that the flow is threedimensional even for an adiabatic ceiling. Such a situation changes the comfort inside the premises.

\section{Conclusion}

Simulation results show that for natural thermal convection, increasing the number of thermal Rayleigh intensifies flow and improves heat transfer. For natural thermosolutal convection, it has been found that the thermal Rayleigh number accelerates the flow and improves both transfers.In the case of mixed convection, we found that the heating temperature and the type of ventilation play a key role in the dynamics of the flow and distribution of thermal fields inside the cavity. The influence of ventilation has also been treated. The results obtained indicated that the $\mathrm{Ra}_{\mathrm{T}}$ numbers have important effects on the temperature distribution

We examined also the air quality inside a threedimensional isothermal room; the three-dimensional flow is stratified for two specific conditions: the ceiling is adiabatic and the ceiling is cooled by the floor above (the case of the floor of a room in a building with several floors). It has been found that the degree of stratification depends on the number of Rayleighs. For the condition of a cooling ceiling, the structure of the three-dimensional flow has been modified and causes transversal recirculations towards the sides and downwards.

\section{References}

[1] W.D. Baines \& J.S. Turner, J. Fluid Mech. 37: 51-80 (1969).

[2] A.W. Woods, C.P. Caulfield, J.C. Phillips, J. Fluid Mechanics 495: 119-133 (2003).

[3] D. Allano, A. Danlos, B. Patte-Rouland, M. Gonzalez, P. Paranthoën, Congrès SFT08, Toulouse, Mai (2008).

[4] H.E. Papanicolau, Y. Jaluria, Numer. Heat Transf. A 18: 427-461 (1990).

[5] E. Papanicolaou, Y. Jaluria, Numer. Heat Transf. A, 23:463-484 (1993).

[6] T.H. Hsu, S.G. Wang, Numer. Heat Transf. Part A 38: 627-652 (2000).

[7] A. Raji, M. Hasnaoui, Rev. Gen. Therm., 37: 874-884 (1988).

[8] M.T. Bhoite, G.S.V.L. Narasimham, and M.V.K. Murthy, Int. J. Therm. Sci. 44, 125 (2005).

[9] V.V. Calmidi and R.L. Mahajan, Int. J. Heat and Fluid Flow 19: 358 (1998).

[10] S. Singh, M.A.R., Numer. Heat Tr. A-Appl., 44: 233253 (2003).

[11] Fluent Inc. FLUENT 6·.3.26 User's guide. (Fluent Inc.) (2006).

[12] W. Rodi, AIAA, 29th Aerospace Sciences Meeting, Reno, Nevada, (1991).

[13] R.J. Krane and J. Lessee. Proc. Isr ASMEJSME Thermal Engineering Joint Conf. 1: 323-329 (1983).

[14] S. Morsli, M. Boussoufi, A.Sabeur, M. El Ganaoui, R. Bennacer, Int. J. Numer. Methods for Heat \& Fluid Flow, 28.(1): 188-205 (2018). 